\title{
Diabetische Nephropathie - Diagnostik
}

\section{Hader ${ }^{1}$}

J. Plum²

\section{Diabetic nephropathy - diagnostic}

\section{Definition}

Der Begriff „Diabetische Nephropathie“ beinhaltet alle renalen Läsionen, die bei Patienten mit Diabetes mellitus auftreten können, wie die (diffuse oder noduläre) Glomerulosklerose, chronisch interstitielle Nephritis, Papillennekrosen sowie verschiedene tubuläre Läsionen. Die „diffuse diabetische Glomerulosklerose“ ist die häufigste Läsion und findet sich bei den meisten Diabetikern mit Nephropathie, unabhängig von der klinischen Manifestationen einer renalen Beteiligung. Die „noduläre Glomerulosklerose (Тyp Kimmelstiel-Wilson)“ ist spezifisch für einen Diabetes mellitus.

\section{Epidemiologie}

Nach Studien liegt in Deutschland die Diabetes Prävalenz bei ca. 4,5\%, auf Grundlage der WHO- bzw. ADA-Kriterien wird sie auf ca. 8,5-9\% geschätzt. Patienten mit Typ-1-Diabetes und solche, deren Typ-2-Diabetes bis zum mittleren Alter manifest wird, entwickeln in 30-40\% eine diabetische Nephropathie $(1,2,10)$. 75\% der Typ-1Diabetiker mit Mikroalbuminurie erreichen ohne adäquate Therapie nach 10-15 Jahren das Stadium der manifesten Proteinurie. Nach den Daten der „Quasi Niere Studie 2000“ liegt bei 21\% der dialysepflichtigen Patienten in Deutschland der Niereninsuffizienz ein Diabetes (5\% Typ 1, 16\% Typ 2) ursächlich zugrunde. Einige Dialysezentren (z.B. unterer Neckar) berichten aber bei allgemein zunehmender Prävalenz der diabetischen Nephropathie von über 50\% Diabetikern (v.a. Typ 2) unter den Dialyse-Patienten (11). Die Mortalität von Diabetikern mit diabetischer Nephropathie ist in allen Stadien der Nephropathie infolge kardialer, zerebraler, vaskulärer und infektiöser Komplikationen sehr hoch. Für ältere Patienten mit Typ-2-Diabetes scheint die Wahrscheinlichkeit, an einer KHK zu sterben größer zu sein, als die Wahrscheinlichkeit eine terminale Niereninsuffizienz zu entwickeln. Durch die verbesserte KHK Therapie steigt deshalb der Anteil der Patienten mit Typ-2-Diabetes, der eine Niereninsuffizienz entwickelt.

\section{Pathologie und Pathogenese}

Drei typische Veränderungen werden in den Glomeruli bei Patienten mit diabetischer Nephropathie gefunden:
1. Mesangiale Proliferation
2. Glomeruläre Basalmembranverdickung
3. Glomeruläre Sklerose.

$\mathrm{Zu}$ den zum Teil tierexperimentell belegten Ursache für diesen progressiv verlaufenden Prozess gehören der direkte Einfluss von Advanced Glycosylation End Products (AGE's), eine Aktivierung der Proteinkinase $C$, die vermehrte Expression von TGF- $\beta$ als Wachstumsfaktor und genetische Faktoren wie u.a. ACE Polymorphismus $(12,13)$, Veränderung des Natrium-ProtonenAntiporters oder Veränderungen des Apolipoproteins E. Advanced glycosilated endproducts (AGE) entstehen durch kovalente Bindung der Glukose an Proteine. Sie sind im Blut diabeteskranker Patienten erhöht. Die klinische Bedeutung ist noch immer unklar. AGE wirken über eine Verdickung der Basalmembran mit Einbau von Plasmaproteinen sowie über eine Steigerung der Kollagen-Synthese schädigend auf die Nierenfunktion. Dem arteriellen Blutdruck kommt ein wesentlicher modulierender Effekt für die Propagierung der o.g. Veränderungen und die Verstärkung der Risikofaktoren zu (15). Die glomeruläre Hyperfiltration hat eine eigenständige Bedeutung, letztere wird aber durch den arteriellen Blutdruck wesentlich mitbestimmt (16). Vergleichbares gilt für die Hyperglykämie. Bei Patienten mit einem erhöhten $\mathrm{HbA}_{1 \mathrm{c}}$-Wert verschlechtert sich die Nierenfunktion schneller als bei Patienten mit niedrigerem $\mathrm{HbA}_{1 \mathrm{c}}$ (17). Der Zusammenhang zwischen $\mathrm{HbA}_{1 \mathrm{c}}$ und Nierenfunktion gilt vor allem für Typ-1-Diabetiker, bei Typ-2-Diabetikern scheint diese Beziehung weniger stark ausgeprägt. Eine Albuminurie kann Folge aber auch Progressionsfaktor einer diabetischen Nephropathie sein. Die Albuminausscheidung über den Urin hängt u.a. von dem glomerulären transmembranösen Druck, der Ladung

${ }^{1}$ Kliniken St. Antonius, Wuppertal

${ }^{2}$ Heinrich Heine Universität, Klinik für Nephrologie und Rheumatologie (Direktor: Prof. Dr. Grabensee), Düsseldorf

Dr. med. Claus Hader - Kliniken St. Antonius Zentrum Innere Medizin (Standort Marienheim) · Hardtstr. 46 . 42107 Wuppertal · Tel.: 0202/299-4910 · Fax: 0202/299-4015 · E-Mail: claushader@hotmail.com 
der Basalmembran, ihrer Porengröße und der tubulären Albuminrückresorption ab. Eine Proteinurie fördert die Entwicklung einer Glomerulosklerose. Einerseits werden zytotoxische Wirkungen beschrieben, andererseits werden Mesangialzellen und auch tubulointerstitielle Zellen aktiviert und eine chronische Entzündungsreaktion unterhalten.

Neben den Risikofaktoren der hämodynamischen und metabolischen Veränderungen besteht offensichtlich auch eine genetische Prädisposition für die Entstehung der Nephropathie (18). Dafür könnte sprechen, dass in Amerika die Inzidenz und Schwere der diabetischen Nephropathie in der schwarzen Bevölkerung gegenüber der kaukasischen Rasse etwa 3-6mal erhöht ist (19).

\section{Stadieneinteillung nach Mogensen}

Eine verbreitete Einteilung der diabetischen Nephropathie ist die Klassifikation nach Mogensen (Tab.1). Die klinische Relevanz dieser Stadieneinteilung ist gering, da nur die Stadien III-V klinisch erfassbar sind. Dem Stadium III kommt eine besondere Bedeutung zu, da es das erste klinisch fassbare und gleichzeitig letzte potenziell reversible Stadium der diabetischen Nephropathie ist.

lkurzgefasst: Patienten mit Typ-1-Diabetes und solche, deren Typ-2-Diabetes bis zum mittleren Alter manifest wird, entwickeln in 30-40\% eine diabetische Nephropathie. Bei 21\% der dialysepflichtigen Patienten in Deutschland ist die Ursache für die Niereninsuffizienz ein Diabetes mellitus. Mit einem Anstieg der Zahl ist zu rechnen. Die Mortalität von Diabetikern mit einer diabetischen Nephropathie ist in allen Stadien der Nephropathie sehr hoch. Eine verbreitete Stadieneinteilung ist die Klassifikation nach Mogensen.

\section{Diagnostik der Nephropathie}

Albuminurie
Albumin wird in geringen Mengen glomerulär filt
weitgehend wieder rückresorbiert. Eine gering
minausscheidung, die sogenannte Mikroalbun
mit den üblichen Eiweißteststreifen nachweisb
der Albuminausscheidung kann neben einer
durch zahlreiche extrarenale Einflussfaktoren v
- Sport oder starke körperliche Betätigung
- Infekte der ableitenden Harnwege
- Klinisch manifeste Herzinsuffizienz
- Blutzuckerentgleisung
- Blutdruckentgleisung
- Fieber
- Operationen (5)

Lässt sich nach Beseitigung der Ursache keine Albuminurie mehr nachweisen, handelt es sich um eine passagere Albuminurie.

Screening: Zum Ausschluss einer diabetischen Nephropathie genügt gemäß den Empfehlungen der Deutschen Diabetesgesellschaft (DDG) nach dem 12. Lebensjahr ein jährliches Scree-
Tab.1 Diabetische Nephropathie: Stadieneinteilung nach Mogensen.

\begin{tabular}{|c|c|c|}
\hline Stadium & $\begin{array}{l}\text { Klinische und histologische } \\
\text { Befunde }\end{array}$ & $\begin{array}{l}\text { Verlauf und } \\
\text { Prognose }\end{array}$ \\
\hline $\begin{array}{l}\text { Stadium I: } \\
\text { Hypertrophie-Hyper- } \\
\text { funktions-Stadium }\end{array}$ & $\begin{array}{l}\text { Erhöhte glomeruläre Filtrati- } \\
\text { onsleistung. } \\
\text { Sonographie: Vergrößerte } \\
\text { Nieren }\end{array}$ & $\begin{array}{l}\text { Eine gute Stoff- } \\
\text { wechseleinstellung } \\
\text { bewirkt einen Rück- } \\
\text { gang der Verände- } \\
\text { rungen, ohne sie zu } \\
\text { normalisieren }\end{array}$ \\
\hline $\begin{array}{l}\text { Stadium II: } \\
\text { Latente Nephropathie }\end{array}$ & $\begin{array}{l}\text { Histologische Veränderun- } \\
\text { gen können vorhanden sein: } \\
\text { Verdickung der Basalmemb- } \\
\text { ran, mesangiale Expansion. }\end{array}$ & $\begin{array}{l}\text { Vollständige Rever- } \\
\text { sibilität fraglich }\end{array}$ \\
\hline $\begin{array}{l}\text { Stadium III: } \\
\text { Inzipiente Nephropa- } \\
\text { thie / Frühestes klini- } \\
\text { sches } \\
\text { Nephropathiestadium }\end{array}$ & $\begin{array}{l}\text { Konstant erhöhte Albumi- } \\
\text { nexkretionsrate ( } 20- \\
200 \mu \mathrm{g} / \mathrm{min} \text { Albumin). } \\
\text { Erhöhte Prävalenz für Blut- } \\
\text { hochdruck (ca. } 40 \% \text { ) (4), } \\
\text { Hyperlipoproteinämie, pro- } \\
\text { liferative Retinopathie und } \\
\text { autonome Neuropathie }\end{array}$ & $\begin{array}{l}\text { Reversibilität frag- } \\
\text { lich } \\
\text { 20fach erhöhtes } \\
\text { Risiko, eine mani- } \\
\text { feste diabetische } \\
\text { Nephropathie zu } \\
\text { entwickeln }\end{array}$ \\
\hline $\begin{array}{l}\text { Stadium IV: } \\
\text { Manifeste diabetische } \\
\text { Nephropathie }\end{array}$ & $\begin{array}{l}\text { Glomerulosklerose } \\
\text { Konstante unselektive Pro- } \\
\text { teinurie }>0,5 \mathrm{~g} / 24 \mathrm{~h} \text {, Abfall } \\
\text { der glomerulären Filtration. }\end{array}$ & $\begin{array}{l}\text { Kalkulierbare Abfall- } \\
\text { rate der Nierenfunk- } \\
\text { tion, im Mittel um } \\
1 \mathrm{ml} / \text { min pro } \\
\text { Monat. Durch The- } \\
\text { rapie ist eine deutli- } \\
\text { che Verlangsamung } \\
\text { möglich. }\end{array}$ \\
\hline $\begin{array}{l}\text { Stadium V: } \\
\text { Niereninsuffizienz }\end{array}$ & $\begin{array}{l}\text { Fortschreitender Abfall der } \\
\text { Nierenfunktion.(GFR<40ml/ } \\
\text { min). Erhöhte Serumkreati- } \\
\text { ninwerte und Serumharn- } \\
\text { stoffwerte. Ggf. } \\
\text { Hyperphosphatämie, Hypo- } \\
\text { kalzämie, renale Azidose, } \\
\text { sekundärer Hyperparathyre- } \\
\text { oidismus und renale Anämie. }\end{array}$ & $\begin{array}{l}\text { Irreversibler Verlust } \\
\text { der Nierenfunktion. } \\
\text { Mitunter entwickelt } \\
\text { sich eine große Pro- } \\
\text { teinurie mit Dyspro- } \\
\text { teinämie und } \\
\text { Hypalbuminämie. } \\
\text { Hohe Letalität }\end{array}$ \\
\hline
\end{tabular}

ning mit dem Nachweis einer physiologischen Albuminausscheidung (Micral-II; Rapitex-Albumin, Mikrobumin-Test) in einer Morgen- oder Spontanurinprobe (normal: <20 mg/l). Mit dem Screening sollte bei Patienten mit Typ-1-Diabetes ab dem 5. Jahr nach Diabetesdiagnose oder bei Kindern mit dem Einsetzen der Pubertät begonnen werden. Patienten mit Diagnosestellung eines Typ-2-Diabetes vor dem 60. Lebensjahr sollte wegen des schleichenden, oft klinisch nicht erkannten Verlaufs bereits bei Diagnosestellung eine Untersuchung erfolgen.

Diagnose: Fällt das Ergebnis der Screening-Untersuchung pathologisch aus, wird zum Nachweis einer diabetischen Nephropathie zweimalig im Abstand von 2-4 Wochen eine persistierend erhöhte Albuminausscheidungsrate im 24 Stunden Urin gefordert (zum Beispiel>30 mg im 24-Stunden-Urin). Zur Bestimmung der Albuminausscheidungsrate sind verschiedene Urinsammelperioden möglich (Tab. 2). Bei Patienten mit Typ-2-Diabetes weist ein mehrfacher Nachweis einer Mikroalbuminurie (zum Beispiel 30$300 \mathrm{mg} / 24$ Stunden) nicht unbedingt auf eine diabetische Nephropathie hin, ist jedoch Ausdruck eines erhöhten Risikos für kardio- und zerebrovaskuläre Ereignisse. Der Nachweis einer Makroalbuminurie (zum Beispiel > $300 \mathrm{mg} / 24$ Stunden) spricht hingegen bei gleichzeitigem Vorliegen einer diabetischen Retinopathie mit hoher Treffsicherheit für eine diabetische Nephropathie. Bei zweifelhaften Befunden müssen andere Nierenerkrankungen 
als Ursache in Erwägung gezogen werden. Insbesondere eine Hämaturie, eine rasch progressive Niereninsuffizienz oder Proteinurie, sonographische Veränderungen der Nieren sowie eine Diabetesdauer $<5$ Jahre bei Typ-1-Diabetes sollten weitere Diagnostik durch einen Nephrologen nach sich ziehen (ggf. Indikation zur Nierenbiopsie Abb.1 und Abb.2) (6).

kurzgefasst: Um eine diabetische Nephropathie auszuschließen, sollte ein Screening auf eine pathologisch erhöhte Albuminausscheidung durchgeführt werden. Der mehrfache Nachweis einer Mikroalbuminurie weist bei Patienten mit Diabetes mellitus Typ 1 auf eine diabetische Nephropathie, bei Patienten mit Diabetes mellitus Typ 2 auf ein erhöhtes Risiko für kardio- und zerebrovaskuläre Ereignisse hin. Eine Makroalbuminurie spricht auch bei Typ-2-Diabetikern - bei gleichzeitig vorliegender Retinopathie - mit hoher Wahrscheinlichkeit für eine diabetische Nephropathie.

\section{Verlaufskontrolle}

Protein- und Albuminurie sind als Verlaufsparameter für den Schweregrad einer diabetischen Nephropathie nicht geeignet, sollten dennoch jährlich bestimmt werden. Bei allen Diabetikern sollte jährlich - bei eingeschränkter Nierenfunktion mindestens 2-4-mal im Jahr - Serumkreatinin- und Serumharnstoffwert bestimmt werden. Bei Patienten mit Nephropathie sollten jährlich Gesamtcholesterin-, HDL- und LDL-Cholesterinspiegel bestimmt, ein Ruhe- und ein Belastungs-EKG durchgeführt sowie regelmäßig Augenhintergrund und angiologischer Status überprüft werden (20).

Um die hohe Morbidität bei fortgeschrittener diabetischer Nephropathie zu senken, müssen alle durch die Niereninsuffizienz und den Diabetes induzierten Komplikationen und Folgeerkrankungen einzeln erfasst und einer entsprechenden Therapie zugeführt werden. Bei steigenden Kreatininwerten ist zu beachten, dass Diabetiker die fortgeschrittene Niereninsuffizienz schlechter tolerieren: Sie entwickeln früher urämische Symptome wie Übelkeit, Erbrechen und Anämie. Eine ausgewogene Diagnostik und Therapie der Folgen der eingeschränkten Nierenleistung ist deshalb wichtig. Erniedrigte Bikarbonatspiegel führen im Verlauf der Erkrankung zu einer progressiven metabolischen Azidose: Dadurch wird der Serumspiegel des Wachstumsfaktors Igf1 (Insulin like growth factor) erniedrigt. Dies fördert die Insulinresistenz und induziert eine katabole Stoffwechselsituation. Außerdem sinkt die Albuminsynthesera-

Tab.2 Mikroalbuminurie: Sammelmöglichkeiten und Grenzen.

\begin{tabular}{ll}
\hline Befristete Urinsammlung: & $20-200 \mu \mathrm{g} / \mathrm{min}$ \\
24-Stunden-Urinsamm/ung: & $30-300 \mathrm{mg} / 24 \mathrm{~h}$ \\
Albumin / Kreatinin Quotient: & für Frauen: $30-300 \mathrm{mg} / \mathrm{g}$ U-Krea \\
& $3,5-35 \mathrm{mg} / \mathrm{mmol}$ U-Krea \\
& für Männer: $20-200 \mathrm{mg} / \mathrm{g}$ U-Krea \\
& $2,5-25 \mathrm{mg} / \mathrm{mmol}$ U-Krea
\end{tabular}

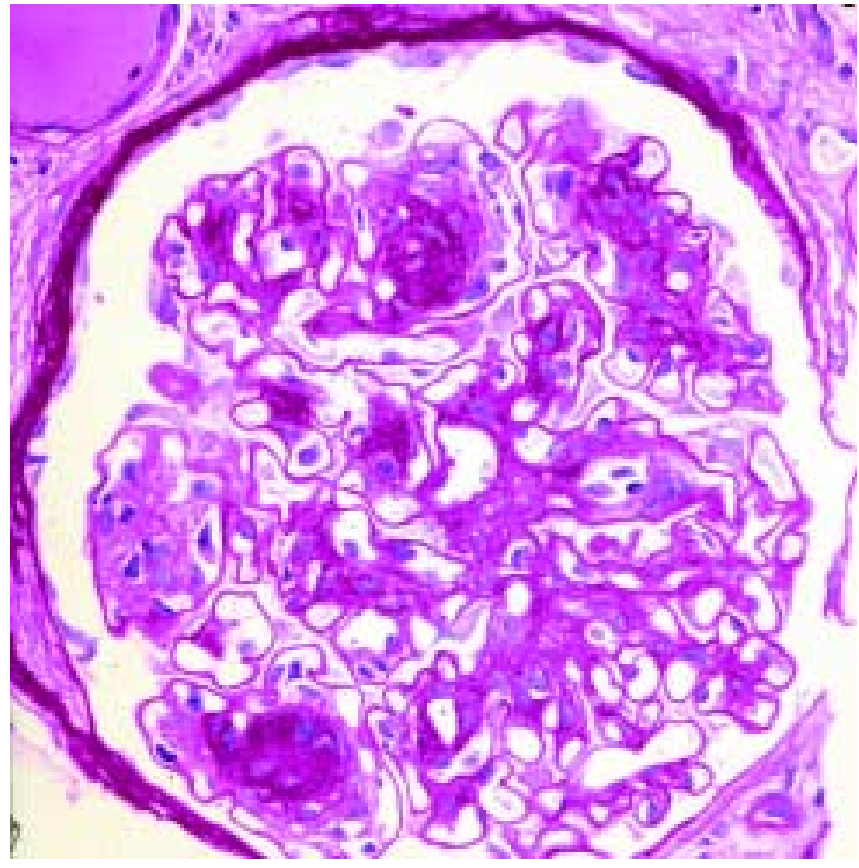

Abb. 1 Mittelschwere teils diffuse, teils noduläre diabetische Glomerulosklerose sowie mittelschwere sog. benigne Nephrosklerose. Es finden sich ausgeprägte herdförmige glomeruläre und tubulointerstitielle Vernarbungen. Der Biopsiebefund stammt von einem 71jährigen Patienten mit Diabetes mellitus Typ 2 und großer Proteinurie bei kompensierter Niereninsuffizienz (Die pathologische Begutachtung erfolgte durch Prof. Dr. U. Helmchen, Kerninstitut für Pathologie, Universitäts-Krankenhaus Eppendorf, Hamburg).

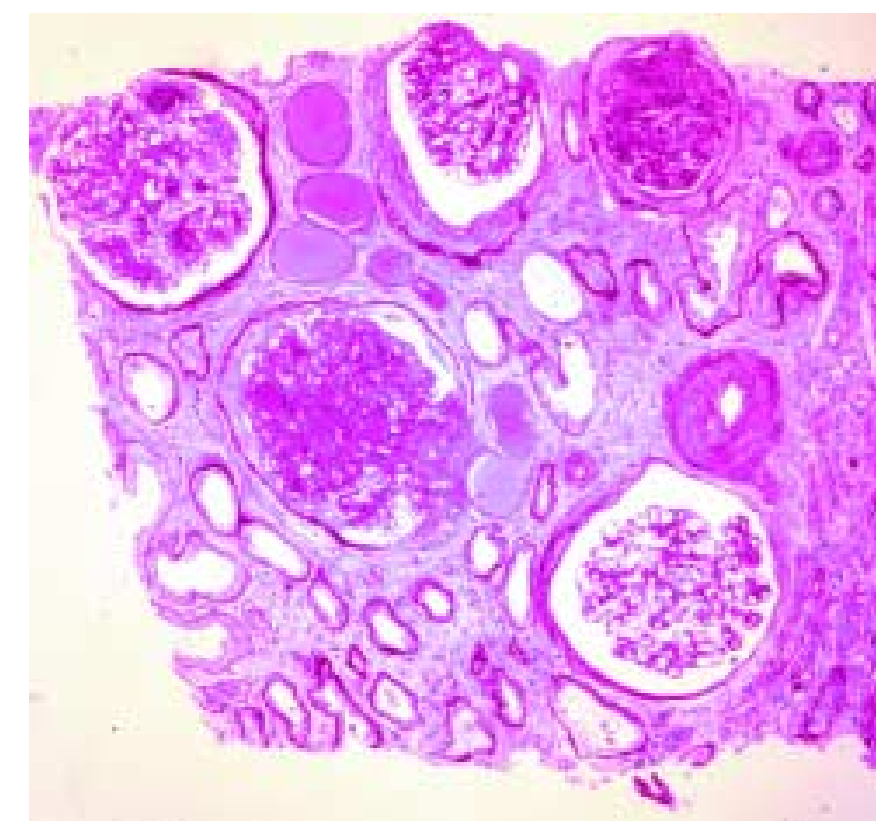

Abb.2 Nierenmark und -rinde mit z.T. vollständig vernarbten Glomeruli. Tubulärer Apparat der Rinde herdförmig atrophisch und umgeben durch ein fibrosiertes und lymphoplasmazellulär infiltriertes Interstitium (Die pathologische Begutachtung erfolgte durch Prof. Dr. U. Helmchen, Kerninstitut für Pathologie, Universitäts-Krankenhaus Eppendorf, Hamburg). 
te, die Entwicklung eines sekundären Hyperparathyreoidismus wird durch Hemmung der Vitamin D-Synthese gefördert. Es wird vermutet, dass hierdurch die Progression einer Arteriosklerose beschleunigt werden kann. Durch verminderte Phosphatelimination kommt es auch zum Anstieg der Serumphosphatwerte und damit konsekutiv zu einem Abfall der Vitamin D-Konzentrationen und sekundär zur Förderung des Hyperparathyreoidismus. Bei erhöhten Phosphatspiegeln findet sich 52\% häufiger eine KHK. Da deutlich erhöhte Phosphatwerte bereits im Stadium der kompensierten Retention gesenkt werden sollten (7), sind Kontrollen des Kalzium-Phosphathaushaltes erforderlich, ggf. ist dann auch eine Bestimmung des Parathormon angezeigt. Eine Blutgasanalyse gehört bei fortgeschrittener Nephropathie zur Standarduntersuchung.

Eine renale Anämie tritt bei Diabetikern früher auf und ist häufig rascher im Verlauf. Sie fördert die linksventrikuläre Hypertrophie und sekundär die linksventrikuläre Dilatation. Sie kann eine Herzinsuffizienz induzieren. Der Blutdruck sinkt tendenziell. Da Empfehlungen zu einer Anhebung des Hämoglobinwertes auf Werte um $12 \mathrm{~g} / \mathrm{dl}$ vorliegen (8), sind Blutbildkontrollen erforderlich. Weiter besteht die Neigung zur Hyperkaliämie bei häufiger auftretendem primärem hyporeninämischen Hypoaldosteronismus. Die Gefahr der Hyperkaliämie ist besonders gegeben unter dem Einsatz kaliumsparender Diuretika. Regelmäßige Elektrolytkontrollen unter Therapie sind insbesondere bei fortgeschrittener Niereninsuffizienz erforderlich.

\section{Iatrogene Schädigungen}

Kontrastmittel: Patienten mit Diabetes mellitus haben ein erhöhtes Risiko, nach Kontrastmittelexposition ein akutes Nierenversagen zu erleiden (9). Kontrastmitteluntersuchungen sind deshalb nur nach strenger Indikationsstellung durchzuführen. Vor Kontrastmitteluntersuchungen ist eine ausreichende Hydrierung erforderlich. Der zusätzliche Einsatz von Diuretika ist nicht zu empfehlen. Für die prophylaktische Gabe vasoaktiver Substanzen wie Theophyllin oder Kalziumantagonisten ist kein Nutzen bewiesen.

Medikamente: Häufig finden sich zahlreiche nephrotoxische Medikamente in den Verordnungen nierenkranker Diabetiker. $\mathrm{Zu}$ nennen sind unter anderem Aminoglykoside, Cotrimoxazol und weitere Antibiotika, Cisplatin, Methotrexat, nichtsteroidale Antiphlogistika, D-Penicillamin, Gold und Fibrate. Die nephrotoxische Wirkung von Arzneimittelkombinationen, insbesondere unter ACE Hemmer Therapie (z.B. Schleifendiuretika plus nichtsteroidale Antiphlogistika plus Antibiotika), multipliziert sich. Der Einsatz eines Röntgenkontrastmittels führt dann nicht selten zu einem akuten Nierenversagen.

Hydratationszustand: Häufig ist aufgrund einer Herzinsuffizienz und einer Neigung zur Wasserretention eine vorübergehende Negativbilanzierung erforderlich. Eine zu starke Verminderung der Flüssigkeitszufuhr, gastrointestinale Flüssigkeitsverluste oder übermäßige Diuretikagabe können jedoch eine Niereninsuffizienz verschlechtern. Es kommt zu einer gesteigerten Vasopressin-Freisetzung, der intraglomeruläre Druck wird erhöht, was möglicherweise die Glomerulosklerose fördert. Die regelmäßige Beurteilung des Hydratationszustandes (klinisch, laborchemisch, ggf. sonographisch oder radiologisch) ist bei einzelnen Patienten erforderlich.

\section{Literatur}

1 Andersen AR, Christiansen JS, Andersen JK, Kreiner S, Deckert T. Diabetic nephropathy in Type 1 (insulin-dependent) diabetes: an epidemiological study. Diabetologia 1983; 25: 496-501

2 Hasslacher C, Ritz E, Wahl P, Michael C. Similar risks of nephropathy in patients with type I or type II diabetes mellitus. Nephrol.Dial.Transplant 1989; 4: 859-863

3 Banholzer P, Haslbeck M, Edelmann E et al. Sonographic changes in the size of the kidneys in type I diabetes as a method of early detection of diabetic nephropathy. Ultraschall Med 1988; 9: 255-259

${ }^{4}$ Baba T, Murabayashi S, Aoyagi K et al. Prevalence of hypertension in diabetes mellitus - its relation to diabetic nephropathy. Tohoku J Exp Med 1985; 145: 167-173

${ }^{5}$ Mogensen CE. Microalbuminuria in prediction and prevention of diabetic nephropathy in insulin-dependent diabetes mellitus patients. J Diabetes Complications 1995; 9: 337-349

${ }^{6}$ Catalano C. De novo diabetes in dialysis patients: when diabetes is not diabetic nephropathy. Nephrol Dial Transplant 1996; 11: 938-941

7 Amann K, Gross ML, London GM, Ritz E. Hyperphosphataemia - a silent killer of patients with renal failure? Nephrol Dial Transplant 1999; 14: 2085-2087

8 Foley RN, Parfrey PS, Harnett JD et al. Impact of hypertension on cardiomyopathy, morbidity and mortality in end-stage renal disease. Kidney Int 1996; 49: 1379-1385

9 Parfrey PS, Griffiths SM, Barrett BJ et al. Contrast material-induced renal failure in patients with diabetes mellitus, renal insufficiency, or both. A prospective controlled study. N Engl J Med 1989; 320: 143-149

10 Ritz E, Orth SR. Nephropathy in patients with Type 2 diabetes mellitus. N Engl J Med 1999; 341: 1127-1133

11 Ritz E. Lippert J, Keller C. Rapider Anstieg der Zahl niereninsuffizienter Typ-2-Diabetiker. Dtsch Med Wochenschr 1996: 121-1247

12 Singh AK, Mo W, Dunea G. Effect of glycated proteins on the matrix of glomerular epithelial cells. J Am Soc Nephrol 1998; 9: 908

13 Wof G, Ziyadeh FN. Molecular mechanismus of diabetic renal hypertrophy. Kidney Int 1999; 56: 393

${ }^{14}$ Kopple JD, Zhu X, Lew NL, Lowrie EG. Body weight-for-height relationships predict mortality in maintenance hemodialysis patients. Kidney Int 1999; 56: 1136-1148

${ }^{15}$ Barzilay J, Warram JH, Bak M. Predisposition to hypertension: Risk factor for nephropathy and hypertension in IDDM. Kidney Int 1997; 41: 723

${ }^{16}$ Rudberg S, Persson B, Dahlquist G. Increased glomerular filtration rate as a predictor of diabetic nephropathy - an 8-year prospective study. Kidney Int 1992; 41: 822

17 Bojestic M, Harnqvist HJ, Hermansson G. Declining incidence of nephropathy in insulin-dependent diabetes mellitus. N Engl J Med 15: 330-1994

18 Krolewski AS, Laffel LM, Krolewski M. Glycosylated hemoglobin and the risk of microalbuminuria in patients with insulin dependent diabetes mellitus. N Engl J Med 1995; 332: 1251-1255

19 Smith ST, Svetkey LP, Dennis VW. Racial differences in the incidence and progression of renal diseases. Kidney Int 1991; 40: 815

${ }^{20}$ Arbeitsgemeinschaft Diabetes und Niere der Deutschen Diabetes Gesellschaft. Entscheidungsbasis für die Behandlung von Patienten mit diabetischer Nephropathie. Diabetes und Stoffwechsel 2000; 9: 31-45 